\title{
On bounds and non-existence in the problem of steady waves with vorticity
}

\author{
Vladimir Kozlov ${ }^{1}$, Nikolay Kuznetsov ${ }^{2}$ and Evgeniy Lokharu ${ }^{1}$ \\ ${ }^{1}$ Department of Mathematics, Linköping University, S-581 83 Linköping, Sweden \\ ${ }^{2}$ Laboratory for Mathematical Modelling of Wave Phenomena, \\ Institute for Problems in Mechanical Engineering, Russian Academy of Sciences, \\ V.O., Bol'shoy pr. 61, St. Petersburg 199178, Russian Federation \\ E-mail: vladimir.kozlov@liu.se; nikolay.g.kuznetsov@gmail.com; \\ evgeniy.lokharu@liu.se
}

\begin{abstract}
For the problem describing steady, gravity waves with vorticity on a twodimensional, unidirectional flow of finite depth the following results are obtained. (i) Bounds for the free-surface profile and for Bernoulli's constant. (ii) If only one parallel shear flow exists for a given value of Bernoulli's constant, then there are no wave solutions provided the vorticity distribution is subject to a certain condition.
\end{abstract}

Keywords: surface gravity waves, waves/free-surface flows

\section{Introduction}

We consider the two-dimensional nonlinear problem describing steady waves in a horizontal open channel of uniform rectangular cross-section. The motion of an inviscid, incompressible, heavy fluid, say, water occupying the channel is supposed to be rotational with a prescribed vorticity distribution. This type of motion commonly occurs in nature as is indicated by observations (see, for example, [15, 16] and references cited therein). The plethora of results obtained for various models describing waves with vorticity is briefly characterised by [13. In that paper, flows with counter-currents as well as unidirectional ones were studied. Further details about the latter flows concerning, in particular, global branches of solutions can be found in $\S 3$ of the survey article by [14].

Here, our aim is to investigate some new properties of the problem about waves on unidirectional flows. In particular, we generalise fundamental bounds for steady irrotational waves found by [8] (see also [6] and [1] for the cases of Stokes and solitary waves, respectively), thus extending results obtained in [11. 


\subsection{Statement of the problem}

Let an open channel of uniform rectangular cross-section be bounded below by a horizontal rigid bottom and let water occupying the channel be bounded above by a free surface not touching the bottom. The water motion is supposed to be twodimensional and rotational; the surface tension is neglected on the free surface of water, where the pressure is constant. These assumptions and the fact that water is incompressible allow us to seek the velocity field in the form $\left(\psi_{y},-\psi_{x}\right)$, where $\psi(x, y)$ is referred to as the stream function. The vorticity distribution $\omega$ is supposed to be a prescribed continuous function depending on $\psi$.

The non-dimensional variables proposed by [7] (see Appendix A in [13] for details of scaling) are used here. In particular, lengths and velocities are scaled to $\left(Q^{2} / g\right)^{1 / 3}$ (the depth of the critical uniform stream in the irrotational case) and $(Q g)^{1 / 3}$, respectively; $Q$ and $g$ are the dimensional quantities for the rate of flow and the gravity acceleration, respectively.

In appropriate Cartesian coordinates $(x, y)$, the bottom coincides with the $x$ axis and gravity acts in the negative $y$-direction. We choose the frame of reference so that the velocity field is time-independent as well as the unknown free-surface profile. The latter is assumed to be the graph of $y=\eta(x), x \in \mathbb{R}$, where $\eta$ is a positive continuous function, and so the longitudinal section of the water domain is $D=\{x \in \mathbb{R}, 0<y<\eta(x)\}$.

The following free-boundary problem for $\psi$ and $\eta$ that describes all kinds of waves has long been known (cf. [7]):

$$
\begin{aligned}
& \psi_{x x}+\psi_{y y}+\omega(\psi)=0, \quad(x, y) \in D ; \\
& \psi(x, 0)=0, \quad x \in \mathbb{R} ; \\
& \psi(x, \eta(x))=1, \quad x \in \mathbb{R} ; \\
& |\nabla \psi(x, \eta(x))|^{2}+2 \eta(x)=3 r, \quad x \in \mathbb{R} .
\end{aligned}
$$

In condition (4) (Bernoulli's equation), $r$ is a constant considered as the problem's parameter and referred to as Bernoulli's constant/the total head. In what follows, we suppose that $\psi$ is a monotonic function of $y$ for all $x \in \mathbb{R}$ because we are going to study unidirectional flows.

\subsection{Main results}

Prior to formulating our results we list some auxiliary facts required in what follows.

By a stream (shear-flow) solution of problem (1)-(4) we mean a pair $(u(y), d)$ (the constant depth of flow is given by $d$ ) such that the following relations hold:

$$
u^{\prime \prime}+\omega(u)=0 \quad \text { on }(0, d), \quad u(0)=0, \quad u(d)=1, \quad\left|u^{\prime}(d)\right|^{2}+2 d=3 r,
$$


here the prime denotes the differentiation with respect to $y$. A detailed study of these solutions, in particular, those describing flows with counter-currents is given in [10. The set of unidirectional solutions of the first three relations (5) is parameterised by $s=u^{\prime}(0)$; it is greater than or equal to $s_{0}=\sqrt{2 \max _{0 \leq \tau \leq 1} \Omega(\tau)}$ as follows from the following expressions for $u$ and $d$ (implicit and explicit, respectively):

$$
y=\int_{0}^{u} \frac{\mathrm{d} \tau}{\sqrt{s^{2}-2 \Omega(\tau)}} \text { and } d=\int_{0}^{1} \frac{\mathrm{d} \tau}{\sqrt{s^{2}-2 \Omega(\tau)}}, \quad \text { where } \Omega(\tau)=\int_{0}^{\tau} \omega(t) \mathrm{d} t .
$$

(Note that $\Omega$ belongs to $C^{1}([0,1])$ ) ) It is clear that $d[=d(s)]$ decreases strictly monotonically and tends to zero as $s \rightarrow+\infty$, whereas $d_{0}=\lim _{s \rightarrow s_{0}+0} d(s)$ can be finite or infinite depending on the behaviour of $\Omega$ on $[0,1]$ (see below).

The first formula (6) and last relation (5) imply the equation

$$
r=\mathcal{R}(s), \quad \text { where } \mathcal{R}(s)=\left[s^{2}-2 \Omega(1)+2 d(s)\right] / 3 .
$$

It is clear that this function has only one minimum, say, $r_{c}>0$ attained at some $s_{c}>s_{0}$. If $d_{0}=+\infty$ and $r>r_{c}$, then (7) has two solutions $s_{+}$and $s_{-}$such that $s_{0}<s_{+}<s_{c}<s_{-}$. Substituting $s_{+}$and $s_{-}$into (6), one obtains the stream solutions $\left(u_{+}, d_{+}\right)$and $\left(u_{-}, d_{-}\right)$, respectively. The shear flows described by these solutions are analogous to the uniform sub- and supercritical flows existing in the irrotational case. If $d_{0}<+\infty$, then both $s_{+}$and $s_{-}$, and consequently, the corresponding stream solutions exist only for $r \in\left(r_{c}, r_{0}\right)$, where $r_{0}=\mathcal{R}\left(s_{0}\right)$. If $r>r_{0}$, then only $s_{-}$exists and defines $\left(u_{-}, d_{-}\right)$.

In order to describe how $d_{0}$ and the corresponding stream solution depend on the vorticity distribution [10] considered the following three options:

(i) $\max _{0 \leq \tau \leq 1} \Omega(\tau)$ is attained either at an inner point of $(0,1)$ or at one (or both) of the end-points. In the latter case, either $\omega(1)=0$ when $\Omega(1)>\Omega(\tau)$ for $\tau \in(0,1)$ or $\omega(0)=0$ when $\Omega(0)>\Omega(\tau)$ for $\tau \in(0,1)$ (or both of these conditions hold simultaneously).

(ii) $\Omega(0)>\Omega(\tau)$ for $\tau \in(0,1]$ and $\omega(0)<0$.

(iii) $\Omega(\tau)<\Omega(1)$ for $\tau \in(0,1)$ and $\omega(1)>0$. Moreover, if $\Omega(1)=0$, then $\omega(0)<0$ and $\omega(1)>0$ must hold simultaneously.

Conditions (i)-(iii) define three disjoint sets of vorticity distributions whose union is the whole set of distributions continuous on $[0,1]$. It occurs that conditions (i) imply that $d_{0}=+\infty$, whereas conditions (ii) and (iii) yield that $d_{0}$ is finite. The last two conditions have the following consequences for solutions of problem (5): $u^{\prime}\left(d_{0}\right)=0$ under conditions (iii), whereas (ii) implies that $u^{\prime}(0)=0$ and $u^{\prime}\left(d_{0}\right) \neq 0$.

In order to formulate our result about fundamental bounds for $\hat{\eta}=\sup _{x \in \mathbb{R}} \eta(x)$ and $\check{\eta}=\inf _{x \in \mathbb{R}} \eta(x)$ we have to impose some restrictions on $\psi$ and $\eta$. 
Conditions (A). The function $\psi$ belongs to $C^{1, \alpha}(\bar{D})$; that is, $|\psi|,\left|\psi_{x}\right|$ and $\left|\psi_{y}\right|$ are bounded on $\bar{D}$, whereas the derivatives satisfy the Hölder condition there. Moreover, $\psi_{y}(x, y) \geq \delta$ on $\bar{D}$ for some $\delta>0$. The function $\eta$ belongs to $C_{l o c}^{0, \alpha}(\mathbb{R})$.

TheOREM 1. Let for some $r>0$ problem (11)-(4) have a non-stream solution $(\psi, \eta)$ satisfying conditions (A). Then the following two assertions hold.

(1) $r>r_{c}$ and $\eta(x)>d_{-}$for all $x \in \mathbb{R}$.

(2) If $r$ belongs to $\left(r_{c}, r_{0}\right)$, then the inequalities $\hat{\eta} \geq d_{+}>\check{\eta}$ are true. Moreover, the left inequality is also strict provided $\hat{\eta}$ is attained at some point.

Furthermore, if $\omega$ satisfies conditions (iii), then every solution of problem (11) (4) satisfying conditions (A) is a stream solutions provided $r \geq r_{0}$.

We expect that the left inequality in assertion (2) is always strict which is the case for irrotational waves; see 8 , where assertions similar to (1) and (2) were obtained. However, there is no analogue of the last assertion for irrotational waves. On the other hand, it extends the result of 12 about the absence of small-amplitude non-stream solutions (not necessarily unidirectional) when $r=r_{0}, s=s_{0}>0$ and a certain restriction is imposed on vorticity. Besides, Theorem 1 does not cover the case when $r>r_{0}$ and the vorticity distribution satisfies conditions (ii) and we discuss it in $\S 2.3$.

\subsection{The partial hodograph transform}

Since we consider unidirectional flows, it is convenient to reformulate problem (11)-(4) using the partial hodograph transform; that is, mapping the unknown domain $D$ onto the strip $S=\mathbb{R} \times(0,1)$ (cf. [5] and [3]):

$$
\bar{D} \ni(x, y) \mapsto(q, p) \in \bar{S}, \quad \text { where } q=x \text { and } p=\psi(x, y) .
$$

These variables are treated as independent, whereas $y=h(q, p)$ is the new unknown function such that $h_{p}(q, p) \geq \delta^{\prime}>0$ on $\bar{S}$ which follows from conditions (A). A straightforward calculation shows that problem (11)-(4) takes the following form:

$$
\mathcal{D} h=0,(q, p) \in S ; \quad h(q, 0)=0, q \in \mathbb{R} ; \quad\left(1+h_{q}^{2}\right) h_{p}^{-2}+2 h=3 r, p=1, q \in \mathbb{R}
$$

Here $\mathcal{D} h$ stands for

$$
\left[\frac{h_{q}}{h_{p}}\right]_{q}-\left[\frac{1+h_{q}^{2}}{2 h_{p}^{2}}+\Omega(p)\right]_{p}
$$

On the other hand, a solution of this problem allows us to recover $\eta$ using the equality

$$
\eta(x)=h(x, 1), \quad x \in \mathbb{R} .
$$

Therefore, we write $\hat{\eta}$ and $\check{\eta}$ for $\sup _{q \in \mathbb{R}} h(q, 1)$ and $\inf _{q \in \mathbb{R}} h(q, 1)$, respectively, in what follows. Note that $h(q, 1)>0$ because $h(q, 0)=0$ and $h_{p}$ is positive in $S$. In order to recover $\psi$ one has to solve the Dirichlet problem (11)-(3) in the domain whose upper boundary is defined by (9). 


\section{Proof of Theorem 1}

To prove Theorem 1 we reformulate it in terms of problem (8).

TheOREM $1^{\prime}$. Let for some $r>0$ problem (8) have a non-stream solution $h \in C^{1, \alpha}(\bar{S})$ such that $h_{p} \geq \delta^{\prime}$ on $\bar{S}$ for some $\delta^{\prime}>0$. Then the following two assertions hold.

(1) $r>r_{c}$ and $h(q, 1)>d_{-}$for all $q \in \mathbb{R}$.

(2) If $r$ belongs to $\left(r_{c}, r_{0}\right)$, then the inequalities $\hat{\eta} \geq d_{+}>\check{\eta}$ are true.

Moreover, if $\omega$ satisfies conditions (iii) and $r \geq r_{0}$, then every solution of problem (8) belonging to $C^{1, \alpha}(\bar{S})$ is a stream solution.

\subsection{Auxiliary propositions}

The proof of Theorem $1^{\prime}$ is based on two auxiliary propositions. The first of these is maximum principle for an elliptic operator that arises when considering $\mathcal{D} \xi-\mathcal{D} \zeta$, where $\xi$ and $\zeta$ are two different solutions of the first equation (8). In [3] (see pp. 155, 156), it is shown that $u=\xi-\zeta$ satisfies the equation

$$
\mathcal{L} u=\left[a^{(q q)} u_{q}+a^{(q p)} u_{p}\right]_{q}+\left[a^{(p p)} u_{p}+a^{(p q)} u_{q}\right]_{p}=0 \text { in } S,
$$

which is uniformly elliptic provided $\xi$ and $\zeta$ have bounded gradients and $\xi_{p}$ and $\zeta_{p}$ are greater than some positive constant.

Proposition 1. Let $u \in W_{\text {loc }}^{1,2}(\bar{S})$ be bounded in $\bar{S}$. If $\mathcal{L} u=0$ on $S$, where $\mathcal{L}$ has the form (10) and its coefficients are bounded and such that $\mathcal{L}$ is uniformly elliptic on $\bar{S}$ with the ellipticity constant $C_{\mathcal{L}}>0$, then the following equalities hold:

$$
\sup _{S} u(q, p)=\sup _{\mathbb{R}}\{u(q, 0), u(q, 1)\} \text { and } \inf _{S} u(q, p)=\inf _{\mathbb{R}}\{u(q, 0), u(q, 1)\} .
$$

Proof. It is clear that the second equality follows from the first one. Note that the assumptions imposed on $u$ imply its Hölder continuity (see, for example, $\S 8.9$ in [4]). Moreover, the Hölder norm of $u$ over $[t, t+1] \times[0,1]$ is bounded by a constant depending on $C_{\mathcal{L}}$, the Hölder exponent and various bounds (for the coefficients of $\mathcal{L}$ and for $u$ itself), but independent of $t$.

In order to prove the first equality (11) we assume the contrary; that is, $\hat{u}=\sup _{S} u$ is strictly greater than $\sup _{q \in \mathbb{R}}\{u(q, 0), u(q, 1)\}$. Then there exist positive numbers $\epsilon$ and $\ell$ (the latter depends on $\epsilon$, generally speaking) such that

$$
\hat{u}-u(q, p) \geq \epsilon \quad \text { on } \quad S_{\ell}=\{(q, p) \in S: q \in \mathbb{R}, p \in(0, \ell) \cup(1-\ell, 1)\} .
$$

It also exists a sequence $\left\{\left(q_{k}, p_{k}\right)\right\}_{k=1}^{\infty}$ such that $\ell<p_{k}<1-\ell$ and $u\left(q_{k}, p_{k}\right) \rightarrow \hat{u}$.

Let $B_{\rho}(q, p)$ denote the open circle of radius $\rho$ centred at $(q, p)$. Since $\hat{u}-u \geq 0$ satisfies $\mathcal{L}(\hat{u}-u)=0$ in $S$, Harnack's inequality (see Corollary 8.21 in [4]) is applicable 
in every $B_{\rho(\ell)}\left(q_{k}, 1 / 2\right)$, where $\rho(\ell)=(1-\ell) / 2$, and so $\left(q_{k}, p_{k}\right) \in B_{\rho(\ell)}\left(q_{k}, 1 / 2\right)$. Therefore, we have

$$
\sup _{B_{\rho(\ell)}\left(q_{k}, 1 / 2\right)}(\hat{u}-u) \leq C \inf _{B_{\rho(\ell)}\left(q_{k}, 1 / 2\right)}(\hat{u}-u) \leq C\left[\hat{u}-u\left(q_{k}, p_{k}\right)\right] \rightarrow 0 \text { as } k \rightarrow \infty
$$

for some $C>0$ that depends on bounds for the coefficients of $\mathcal{L}, C_{\mathcal{L}}$ and $\ell$. Hence the supremum on the left is arbitrarily small provided $k$ is sufficiently large, but this is incompatible with (12), because $S_{\ell}$ overlaps with $B_{\rho(\ell)}\left(q_{k}, 1 / 2\right)$ for all $k=1, \ldots, \infty$. The obtained contradiction proves the proposition.

It is straightforward to verify that for any $s>s_{0}$ such that $\mathcal{R}(s)=r$ the corresponding stream solution of problem (8), say, $H(p ; s)$ has the following form:

$$
H(p ; s)=\int_{0}^{p} \frac{\mathrm{d} \tau}{\sqrt{s^{2}-2 \Omega(\tau)}} .
$$

If $s=s_{0}$ and either of conditions (ii), (iii) is fulfilled, then this formula gives a continuous function whose derivative is infinite at one of the end-points of $[0,1]$.

The next proposition provides an ersatz of Hopf's lemma for an elliptic equation of the form (10) in $\bar{S}$.

Proposition 2. Let $\mathcal{L}$ be an elliptic operator of the form (10). If $u \in C^{1, \alpha}(\bar{S})$ satisfies the equation $\mathcal{L} u=0$ in $S$ and $\sup _{S} u=\sup _{q \in \mathbb{R}} u(q, 1)$, then for any sequence $\left\{q_{k}\right\}_{k=1}^{\infty}$ such that

$$
u\left(q_{k}, 1\right) \rightarrow \sup _{q \in \mathbb{R}} u(q, 1) \quad \text { as } k \rightarrow \infty
$$

the following relations hold:

$$
u_{q}\left(q_{k}, 1\right) \rightarrow 0 \quad \text { as } k \rightarrow \infty \quad \text { and } \quad \limsup _{k \rightarrow \infty} u_{p}\left(q_{k}, 1\right) \geq 0 .
$$

The similar assertion is true with supremum changed to infimum in the assumption, whereas limsup is replaced by liminf and the inequality sign is opposite in the second relation (15).

If supremum is attained at some point $\left(q_{*}, 1\right)$, then $u_{p}\left(q_{*}, 1\right)>0$; if infimum is attained, the inequality sign is opposite.

Proof. The last assertion for supremum is a consequence of Proposition 1 and Hopf's lemma, whereas for infimum it follows by changing $u$ to $-u$. Therefore, we assume that $\hat{u}=\sup _{S} u=\sup _{q \in \mathbb{R}} u(q, 1)$ and take any sequence $\left\{q_{k}\right\}_{k=1}^{\infty}$ satisfying (14) (without loss of generality, we take it tending to $+\infty$ ).

In order to prove relations (15) we assume the contrary, that is, there exist $\epsilon>0$ such that either $\left|u_{q}\left(q_{k}, 1\right)\right| \geq \epsilon$ or $u_{p}\left(q_{k}, 1\right) \leq-\epsilon$ holds for all sufficiently large $k$. Since $u \in C^{1, \alpha}(\bar{S})$, we have

$$
u\left(q_{k} \pm t, 1\right)=u\left(q_{k}, 1\right) \pm u_{q}\left(q_{k}, 1\right) t+O\left(t^{1+\alpha}\right) \quad \text { as } t \rightarrow+0
$$


which yields that $\hat{u}<u\left(q_{k} \pm t, 1\right)$ when $\pm u_{q}\left(q_{k}, 1\right)>\epsilon$ provided $k$ is sufficiently large and $t>0$ is sufficiently small. However, the last inequality for $\hat{u}$ contradicts its definition. Similarly, we have

$$
u\left(q_{k}, 1-t\right)=u\left(q_{k}, 1\right)-u_{p}\left(q_{k}, 1\right) t+O\left(t^{1+\alpha}\right) \quad \text { as } t \rightarrow+0 .
$$

Then the assumption about $u_{p}\left(q_{k}, 1\right)$ gives that $\hat{u}<u\left(q_{k}, 1-t\right)$ provided $t$ is sufficiently small. This is impossible in view of the definition of $\hat{u}$. Thus, relations (15) are proved.

\subsection{Proof of Theorem $1^{\prime}$}

First we prove that $r \geq r_{c}$ and begin with demonstration that there exists $s>s_{0}$ such that $H(1 ; s)=\check{\eta}$. For this purpose we consider vorticity distributions satisfying conditions (i), (ii) and (iii) separately.

If conditions (i) are fulfilled, then $H(1 ; s)$ decreases monotonically from the positive infinity to zero as $s$ goes from $s_{0}$ to $+\infty$. Hence $H(1 ; s)$ attains the value $\check{\eta}>0$ at some $s \in\left(s_{0},+\infty\right)$.

Since conditions (ii) imply that $s_{0}=0$ and $H_{p}(0 ; s) \rightarrow+\infty$ as $s \rightarrow 0$, we have that the function $h_{p}(q, 0)-H_{p}\left(0 ; s_{*}\right)$ is negative and separated from zero for some $s_{*}>0$. Then $h\left(q_{*}, 1\right)-H\left(1 ; s_{*}\right) \leq 0$ for some $q_{*} \in \mathbb{R}$ because otherwise the function $h(q, p)-H\left(p ; s_{*}\right)$ violates Proposition 1. It follows from the last inequality that $H(1 ; s)=\check{\eta}$ for some $s>s_{*}$ because $H(1 ; s)$ monotonically decreases and tends to zero as $s$ grows, whereas $\check{\eta} \leq h\left(q_{*}, 1\right)$.

Since conditions (iii) imply that $s_{0}>0$ and $H_{p}(1 ; s) \rightarrow+\infty$ as $s \rightarrow s_{0}$, we have that $h_{p}(q, 1)-H_{p}\left(1 ; s_{*}\right)$ is negative and separated from zero for all $q \in \mathbb{R}$ and some $s_{*}>s_{0}$. The latter fact is similar to that obtained from conditions (ii). This allows us to repeat literally the considerations used in that case, thus arriving at the conclusion that $H(1 ; s)=\check{\eta}$ for some $s>s_{*}$.

Let us prove the inequalities $r \geq r_{c}$ and $h(q, 1)>\check{\eta}$ for arbitrary $q$. For this purpose we consider $h(q, p)-H(p ; s)$ with $s>s_{0}$ such that $H(1 ; s)=\check{\eta}$ (the existence of that $s$ was just established). According to Proposition 2 , we have $h_{p}\left(q_{k}, 1\right)-H_{p}(1 ; s) \leq \epsilon_{k}$ for some sequences $\left\{q_{k}\right\}_{k=1}^{\infty}$ and $\left\{\epsilon_{k}\right\}_{k=1}^{\infty}$; the last of these has positive elements and tends to zero. Combining this and Bernoulli's equation for $h$, we get

$$
r=\frac{1}{3}\left[\frac{1+h_{q}^{2}}{h_{p}^{2}}+2 h\right] \geq \frac{1}{3}\left[\frac{1}{\left(H_{p}(1, s)+\epsilon_{k}\right)^{2}}+2 h\left(q_{k}, 1\right)\right] .
$$

Letting $k \rightarrow \infty$ in this inequality and taking into account relation (14) and the definition of $\mathcal{R}(s)$, we obtain that $r \geq \mathcal{R}(s)$, and so $r \geq r_{c}$.

In order to prove the strict inequality $r>r_{c}$, let us assume that problem (8) with $r=r_{c}$ has a non-stream solution $h$. To show that this is impossible we use the 
equality

$$
\begin{gathered}
{[\Phi(1 ; s)-1] \int_{-q_{1}}^{q_{2}} w(q, 1 ; s) \mathrm{d} q+\int_{-q_{1}}^{q_{2}} \int_{0}^{1} \frac{H_{p}^{2} w_{q}^{2}+\left(2 h_{p}+H_{p}\right) w_{p}^{2}}{2 h_{p}^{2}} \mathrm{~d} p \mathrm{~d} q} \\
=-\int_{0}^{1}\left[\frac{h_{q}}{h_{p}} \Phi(p ; s)\right]_{q=-q_{1}}^{q=q_{2}} \mathrm{~d} p .
\end{gathered}
$$

Here $H$ is defined by (13), $w(q, p ; s)=h(q, p)-H(p ; s)$ and $\Phi(p ; s)=\int_{0}^{p} H_{t}^{3}(t ; s) \mathrm{d} t$, whereas $q_{1}, q_{2}>0$. For the derivation of this equality see the proof of Lemma 3.1 in [17, where it appears implicitly. Indeed, the left-hand side of (17) is similar to the expression in his formula (3.1). On the other hand, the right-hand side of (17) corresponds to the last term in the Wheeler's identity (3.3).

Taking $s=s_{c}$ in (17) in which case $\Phi\left(1 ; s_{c}\right)=1$ in view of formula (13) for $H$, we reduce (17) to

$$
\int_{-q_{1}}^{q_{2}} \int_{0}^{1} \frac{H_{p}^{2} w_{q}^{2}+\left(2 h_{p}+H_{p}\right) w_{p}^{2}}{2 h_{p}^{2}} \mathrm{~d} p \mathrm{~d} q=-\int_{0}^{1}\left[\frac{w_{q}}{h_{p}} \Phi\left(p ; s_{c}\right)\right]_{q=-q_{1}}^{q=q_{2}} \mathrm{~d} p .
$$

Then the positive integral on the left converges as $q_{1}, q_{2} \rightarrow+\infty$. This implies that there exists two sequences $\left\{q_{1}^{(k)}\right\}_{k=1}^{\infty},\left\{q_{2}^{(k)}\right\}_{k=1}^{\infty}$ that tend to $+\infty$ as $k \rightarrow \infty$ and yield the following relations:

$$
\int_{0}^{1}\left|\frac{w_{q}\left(q_{j}^{(k)}, p ; s_{c}\right)}{h_{p}\left(q_{j}^{(k)}, p\right)}\right| \Phi\left(p ; s_{c}\right) \mathrm{d} p \rightarrow 0 \quad \text { as } k \rightarrow \infty, \quad j=1,2 .
$$

Therefore, we have

$$
\int_{-\infty}^{\infty} \int_{0}^{1} \frac{H_{p}^{2} w_{q}^{2}+\left(2 h_{p}+H_{p}\right) w_{p}^{2}}{2 h_{p}^{2}} \mathrm{~d} p \mathrm{~d} q=0
$$

according to which the gradient of $w$ vanishes a.e., and this is incompatible with the assumption that $h$ is a non-stream solution.

Let us complete the proof of assertion (1). Since there exists $s$ such that $H(1 ; s)=$ $\check{\eta}$ and consequently $r \geq \mathcal{R}(s)$, we have that $d_{-}=d\left(s_{-}\right)$corresponding to $r$ is less than or equal to $\check{\eta}$. Indeed, the latter is equal to $H(1 ; s)=d(s)$ which decreases monotonically, whereas $s_{-} \geq s$ because $r \geq \mathcal{R}(s)$. Assuming that there exists $q^{\circ}$ such that $d_{-}=h\left(q^{\circ}, 1\right)$, we apply Hopf's lemma which implies that $h_{p}\left(q^{\circ}, 1\right)-H_{p}\left(1 ; s_{-}\right)<$ 0 and this is incompatible with (16), where $q=q^{\circ}$ instead of $q_{k}$. The obtained contradiction proves assertion (1).

Let us turn to assertion (2). Taking $r \in\left(r_{c}, r_{0}\right)$ and assuming that $\check{\eta} \geq d_{+}$(this is contrary to the right inequality in this assertion), we apply equality (17) with $s=s_{+}$, and so $\Phi\left(1 ; s_{+}\right)-1 \geq 0$ now. Therefore, one again obtains a contradiction with the 
assumption that $h$ is a non-stream solution by repeating literally the considerations based on (17) and used above for proving that $r \neq r_{c}$. Thus it is shown that the right inequality holds.

In order to prove the left inequality we again assume the contrary; that is, that $\hat{\eta}<d_{+}$. This implies that there exists $H(p ; s)$ such that $H(1 ; s)=\hat{\eta}$. Since for this $H$ the function $w=h-H$ is less than or equal to zero and its maximum vanishes, Proposition 2 yields that $w\left(q_{k}, 1\right) \rightarrow 0$ as $k \rightarrow \infty$ and $w_{p}\left(q_{k}, 1\right) \geq \epsilon_{k}>0$ for some sequence $\left\{q_{k}\right\}_{k=1}^{\infty}\left(\epsilon_{k}\right.$ tends to zero as $\left.k \rightarrow \infty\right)$. Using this sequence in Bernoulli's equation, we obtain that $r \leq \mathcal{R}(s)$, and so either $\hat{\eta} \leq d_{-}$or $\hat{\eta} \geq d_{+}$. The last inequality contradicts to the assumption made, whereas the former inequality is impossible because $d_{-} \leq \check{\eta}$ (see assertion (1)) and $h$ is a non-stream solution.

Finally, let the vorticity distribution satisfy conditions (iii) and let a non-stream solution of problem (8) corresponding to $r \geq r_{0}$ exist and belong to $C^{1, \alpha}(\bar{S})$. We note again that conditions (iii) imply relations $s_{0}>0$ and $H_{p}(1 ; s) \rightarrow+\infty$ as $s \rightarrow s_{0}$, and so we have that $h_{p}(q, 1)-H_{p}\left(1 ; s_{*}\right)$ is negative and separated from zero for all $q \in \mathbb{R}$ and some $s_{*}>s_{0}$. Then $h(q, 1)-H\left(1 ; s_{*}\right) \leq 0$ for all $q \in \mathbb{R}$ because otherwise the function $h(q, p)-H\left(p ; s_{*}\right)$ violates Proposition 2 . Therefore, the last inequality yields that $H(1 ; s)=\hat{\eta}$ for some $s>s_{0}$. Thus we have that

$$
w(q, p)=h(q, p)-H(p ; s) \leq 0 \text { on } \bar{S} \quad \text { and } \quad \sup _{q \in \mathbb{R}} h(q, 1)-H(1 ; s)=0 .
$$

Applying Proposition 2 to this function, we get a sequence $\left\{q_{k}\right\}_{k=1}^{\infty}$ (generally speaking, other than that in the previous paragraph) such that $w\left(q_{k}, 1\right) \rightarrow 0$ as $k \rightarrow \infty$ and $w_{p}\left(q_{k}, 1\right) \geq \epsilon_{k}>0\left(\epsilon_{k}\right.$ again tends to zero as $\left.k \rightarrow \infty\right)$. As above, combining this and Bernoulli's equation, one arrives at the inequality $r \leq \mathcal{R}(s)$. This inequality is strict when $r_{0}<r$, thus yielding $\hat{\eta} \leq d_{-}$, which is impossible. On the other hand, if $r_{0}=r$, then $r=\mathcal{R}(s)$, and so either $s=s_{0}$ or $\hat{\eta}=d_{-}$, both of which lead to a contradiction.

The proof is complete.

\subsection{Flows with the vorticity distribution satisfying conditions}

\section{(ii)}

First we examine Stokes waves of small amplitude for $r \geq r_{0}$. Our aim is to show that the stream function changes sign within the corresponding flow, and so the same is true for the horizontal component of velocity. For this purpose we apply the description of these waves obtained by 13 and based on the following two assumptions:

(I) A stream solution $(u(y), d)$ satisfying problem (5) with $r>r_{c}$ is supposed to be such that $u^{\prime}(d) \neq 0$.

(II) The dispersion equation $\sigma(\tau)=0$ corresponding to a stream solution satisfying assumption (I) has at least one positive root, say, $\tau_{0}$ such that none of the values $k \tau_{0}$ 
$(k=1,2, \ldots)$ is a root of the dispersion equation. Here

$$
\sigma(\tau)=u^{\prime}(d) \gamma^{\prime}(d, \tau)-\left[u^{\prime}(d)\right]^{-1}+\omega(1)
$$

and $\gamma(y, \tau)$ solves the boundary value problem

$$
-\gamma^{\prime \prime}+\left[\tau^{2}-\omega^{\prime}(u)\right] \gamma=0 \text { on }(0, d), \quad \gamma(0, \tau)=0, \quad \gamma(d, \tau)=1,
$$

If $\omega$ belongs to $C^{2, \alpha}(\mathbb{R})$ with $\alpha \in(0,1)$, then assumptions (I) and (II) guarantee that for all sufficiently small values of the parameter $t$ there exists a solution to problem (11)-(4) that has the following representation:

$$
\begin{aligned}
& \psi(x, y ; t)=u\left(\frac{d y}{\eta(x ; t)}\right)+t \cos \frac{\tau_{0} x}{1+\lambda(t)} W\left(\frac{d y}{\eta(x ; t)}\right)+o(t), \\
& \eta(x ; t)=\frac{d}{1+\lambda(t)}+t \cos \frac{\tau_{0} x}{1+\lambda(t)}+o(t) .
\end{aligned}
$$

Here $\lambda(t) \rightarrow 0$ as $t \rightarrow 0$ and $W$ solves the following problem (see formulae (38) and (39) in [13]):

$$
-W^{\prime \prime}+\left[\tau^{2}-\omega^{\prime}(u)\right] W=d^{-1}\left[y u^{\prime} \tau^{2}+2 \omega(u)\right] \quad \text { on }(0, d), \quad W(0)=W(d)=0,
$$

and $W^{\prime}(d)=d^{-1} u^{\prime}(d)-\left[u^{\prime}(d)\right]^{-1}$.

According to Proposition 3.5 (ii) in [13, for every $r>r_{c}$ there exists a unidirectional flow such that assumption (II) does not hold for the corresponding stream solution. Besides, if $r>r_{0}$, then there also exists at least one stream solution satisfying assumption (II). However, every such solution changes sign on $(0, d)$ and the same is true for the stream function (19) provided $|t|$ is small. These facts still hold when $r=r_{0}$ and the stream solution is such that $s=u^{\prime}(0)>s_{0}$.

Let us consider the remaining case, that is, $r=r_{0}, s=s_{0}$ and $u^{\prime}(d) \neq 0$. (This occurs when the vorticity distribution satisfies conditions (ii).) To demonstrate that the stream function (19) changes sign we have to check that $W^{\prime}(0) \neq 0$. In order to verify this we take $w$ that solves the following problem:

$$
-w^{\prime \prime}+\left[\tau^{2}-\omega^{\prime}(u)\right] w=0 \text { on }(0, d), \quad w(0)=1, \quad w(d)=0 .
$$

Its existence is guaranteed by Lemma 2.2 in [13. Multiplying the first relation (20) by $w$, we integrate the result over $(0, d)$ and then integrate by parts in the same way as in the proof of the cited lemma. This leads to the equality $W^{\prime}(0)=d u^{\prime}(d) w^{\prime}(d)$ which implies the required inequality.

As in the case when $\omega$ satisfies conditions (iii) we conjecture that only stream solutions exist when $r \geq r_{0}$ and conditions (ii) hold for $\omega$ (cf. the last assertion of Theorem $1^{\prime}$ ). However, if a solution exists for some $r>r_{0}$, then we have the following 
proposition saying that in this case $d_{0}$ plays the same role as $d_{+}$in assertion (2) of Theorem $1^{\prime}$.

Proposition 3. Let $\omega$ satisfy conditions (ii). If for some $r>r_{0}$ problem (8) has a solution $h \in C^{1, \alpha}(\bar{S})$, then the inequalities $\hat{\eta} \geq d_{0}>\check{\eta}$ hold for it.

Proof. To prove the left inequality we assume the contrary, that is, $d_{0}>\hat{\eta}$. Then for some $s>s_{0}$ there exists a solution of the form (13) such that $H(1, s)=\hat{\eta}$. Applying Proposition 2 to the function $w=h-H$ in the same way as for proving the inequality $r \geq \mathcal{R}(s)$ in the proof of Theorem $1^{\prime}$, we obtain that $d_{-} \geq \hat{\eta}$, but according to assertion (2) of this theorem we have $\check{\eta} \geq d_{-}$. Hence $h$ is identically equal to $H$ which is impossible. Thus, the left inequality is proved.

It was shown in the proof of Theorem $1^{\prime}$ that $s>s_{0}$ can be found from the equation $H(1 ; s)=\check{\eta}$. This implies the right inequality, thus completing the proof.

\section{Concluding remarks}

We have considered the problem describing steady, rotational, water waves in the case when no counter-currents are present in a flow of finite depth (such flows are referred to as unidirectional). It is shown that for the existence of non-stream solutions the problem's parameter $r$ (Bernoulli's constant/the total head) must be strictly greater than the critical value $r_{c}$ — the unique minimum of the function $\mathcal{R}$ (see formula (7) for its definition). Stream solutions describing shear flows with horizontal free surfaces are a kind of trivial solutions like those describing irrotational uniform flows. Thus, the requirement on $r$ obtained here for waves with vorticity generalises that proved by [8] in the irrotational case when two uniform conjugate flows exist for all $r>r_{c}$ (see formula (13) in 99]). Furthermore, another result obtained for irrotational waves in [8] is shown to be also valid for waves with vorticity. It says that the depths of the pair of conjugate, shear flows serve as bounds for the supremum and infimum of the wave profile on a unidirectional flow.

According to a unified theory of conjugate flows developed by [2], their important feature (apart of being unidirectional) is that they are transcritical. This means that one flow is supercritical, whereas the other one is subcritical; that is, the former's (letter's) depth is less (greater) than that of the critical flow corresponding to $r_{c}$. (For a given value of the rate of flow this relates to the opposite relationship between properly defined values of the flow velocity.) In his paper (see also references cited therein), [2] characterised the existence of conjugate flows as a common feature for many hydrodynamic models and emphasised that it 'is crucial to the understanding of observed wave phenomena'.

In this paper the last statement finds the following confirmation. It occurs that along with $r_{c}$ another critical value $r_{0}$ exists in $\left(r_{c},+\infty\right)$ provided the vorticity distribution satisfies either of conditions (ii) and (iii) formulated in $\S 1.2$. It is known 
that for $r>r_{0}$ only the supercritical shear flow is unidirectional, whereas all other shear flows corresponding to these values of $r$ (at least one such flow exists for every $r>r_{0}$ ) have counter-currents (see [10]). Here we proved that only stream solutions describe unidirectional flows when $r \geq r_{0}$ and conditions (iii) hold for the vorticity distribution. This, in particular, implies that the presence of two conjugate flows is essential for the existence of solitary waves supported by a supercritical shear flow. In the case when the vorticity distribution satisfies conditions (ii) and $r \geq r_{0}$ we demonstrate that all known flows with waves (these are Stokes waves of small amplitude; see [12]) have counter-currents. Therefore, it is reasonable to conjecture that there are no unidirectional flows with waves for $r \geq r_{0}$.

There is another reason to refer to $r_{0}$ as the critical value of the second kind. Indeed, it is shown in 12 that no steady water waves of small amplitude are supported by a shear flow with a still free surface.

Acknowledgements. V. K. and E. L. were supported by the Swedish Research Council (VR). N. K. acknowledges the support from G. S. Magnuson's Foundation of the Royal Swedish Academy of Sciences and Linköping University.

\section{References}

[1] Amick, C. J., Toland, J. F. 1981 On solitary waves of finite amplitude. Arch. Ration. Mech. Anal. 76, 9-95.

[2] Benjamin, T. B. 1971 A unified theory of conjugate flows. Phil. Trans. Roy. Soc. Lond. A 269, 587-643.

[3] Constantin, A., Strauss, W. 2011 Periodic travelling gravity water waves with discontinuous vorticity. Arch. Ration. Mech. Anal. 202, 133-175.

[4] Gilbarg, D., Trudinger, N. S. 2001 Elliptic Partial Differential Equations of Second Order. Springer-Verlag.

[5] Groves, M. D., WAhlÉn, E. 2008 Small-amplitude Stokes and solitary gravity water waves with an arbitrary distribution of vorticity. Physica D 237, 1530-1538.

[6] Keady, G., Norbury, J. 1975 Water waves and conjugate streams. J. Fluid Mech. 70, 663-671.

[7] Keady, G., Norbury, J. 1978 Waves and conjugate streams with vorticity. Mathematika 25, 129-150.

[8] Kozlov, V., Kuznetsov, N. 2009 Fundamental bounds for steady water waves. Math. Ann. 345, 643-655.

[9] Kozlov, V., Kuznetsov, N. 2010 The Benjamin-Lighthill conjecture for near-critical values of Bernoulli's constant. Arch. Rat. Mech. Anal. 197, 433-488.

[10] Kozlov, V., Kuznetsov, N. 2011 Steady free-surface vortical flows parallel to the horizontal bottom. Quart. J. Mech. Appl. Math. 64, 371-399. 
[11] Kozlov, V., Kuznetsov, N. 2012 Bounds for steady water waves with vorticity. J. Differential Equations 252, 663-691.

[12] Kozlov, V., Kuznetsov, N. 2013 No steady water waves of small amplitude are supported by a shear flow with a still free surface. J. Fluid Mech. 717, 523-534.

[13] Kozlov, V., Kuznetsov, N. 2014 Dispersion equation for water waves with vorticity and Stokes waves on flows with counter-currents. Arch. Ration. Mech. Anal. 214(1) DOI 10.1007/s00205-014-0787-0.

[14] Strauss, W. 2010 Steady water waves. Bull. Amer. Math. Soc. 47, 671-694.

[15] Swan, C., Cummins, I. \& James, R. 2001 An experimental study of two-dimensional surface water waves propagating in depth-varying currents. J. Fluid Mech. 428, 273304.

[16] Thomas, G. P. 1990 Wave-current interactions: an experimental and numerical study. J. Fluid Mech. 216, 505-536.

[17] Wheeler, M.H. 2014 The Froude number for solitary water waves with vorticity. Preprint available online at http://arXiv.org/abs/1405.1083 